\title{
Monitoreo hemodinámico utilizando variabilidad del volumen sistólico como parámetro para guiar el manejo de líquidos en pacientes sépticos
}

\section{Hemodynamic monitoring using the variability of the systolic volume as a parameter to guide the fluid management in septic patients}

Autores: Víctor Aquino Millán ${ }^{1}$, Hugo Bianco Cáceres², Ariel Fretes Ruiz ${ }^{3}$, Belinda Figueredo ${ }^{4}$, Fernando Ferreira Marecos ${ }^{5}$

\author{
Artículo recibido: 11 febrero $2014 \quad$ Artículo aceptado: 23 febrero 2014
}

Resumen: se presenta una serie de casos donde se compara la variabilidad del volumen sistólico con las variables estáticas habituales en la reanimación con fluidos de pacientes sépticos.

Palabras claves: variabilidad del volumen sistólico, sepsis, hidratación parenteral

\begin{abstract}
: we report a case series where the variability of the systolic volume is compared to the usual static variables of the fluid resuscitation of septic patients.
\end{abstract}

Keywords: systolic volume variability, sepsis, parenteral hydration

\section{Introducción}

La clave en el tratamiento inicial de la sepsis es el manejo de los líquidos corporales y conocer la cantidad exacta de fluidos a administrar es un desafío en cada paciente. ${ }^{1,2,3}$ La precisión de las variables estáticas como objetivo en la reanimación, tales como la frecuencia cardiaca (FC), tensión arterial media (TAM) y presión venosa central (PVC), pareciera ser limitada en comparación a las dinámicas como la variabilidad del volumen sistólico (VVS). ${ }^{4,5}$ El uso de dichos elementos nos orientaría con certeza hacia el manejo óptimo de la volemia. ${ }^{6,7}$

\section{Objetivo}

Determinar el nivel de volemia en pacientes con sepsis severa luego de la resucitación inicial utilizando la VVS para dicho efecto.

\footnotetext{
${ }^{1}$ Médico de guardia. Unidad de Terapia Intensiva Adultos, Hospital de Clínicas. FCM-UNA

${ }^{2}$ Jefe de Servicio. Unidad de Terapia Intensiva Adultos, Hospital de Clínicas. FCM-UNA

${ }^{3}$ Jefe de Servicio Urgencias. Hospital de Clínicas. FCM-UNA

${ }^{4}$ Sub jefa de Servicio. Unidad de Terapia Intensiva Adultos, Hospital de Clínicas. FCM-UNA

${ }^{5}$ Jefe de sala. Unidad de Terapia Intensiva Adultos, Hospital de Clínicas. FCM-UNA

\section{Autor correspondiente:}

Dr. Víctor Aquino Millán. Especialista en Medicina Interna

Dirección: Guayaibi entre Ettiene y Juana de Lara. Zona Norte. Fernando de la Mora, Paraguay

Teléfono: 595981179008

Correo electrónico: victoraquino81@gmail.com
} 


\section{Materiales y métodos}

Diseño observacional, retrospectivo, de corte transverso. Se incluyeron pacientes en el periodo comprendido entre marzo y junio del 2013, sin patología de base, que ingresaron a la Unidad de terapia intensiva de adultos del Hospital de Clínicas, FCM-U.N.A. con sepsis severa y requerimiento de asistencia respiratoria mecánica. Luego de haberse realizado la resucitación inicial siguiendo las guías de la campaña sobreviviendo la sepsis ${ }^{4}$, se midió la VVS con el Clinic Platform EV 1000 mediante el sensor Floctrac como parámetro para valorar el volumen del líquido intravascular teniendo como punto de corte para déficit de volumen una VVS $>13 \%{ }^{3,6}$

\section{Resultados}

Se incluyeron 3 pacientes de sexo masculino que ingresaron a terapia intensiva, con edad media 29,3 años (rango 16-43), con diagnóstico de sepsis severa a punto de partida abdominal. Luego de la resucitación inicial presentaron las siguientes variables promedio: FC 94,3 latidos x minuto (rango 73-110), TAM 82,3 mm $\mathrm{Hg}$ (rango 70-95), PVC 15,3 $\mathrm{cm} \mathrm{H}_{2} \mathrm{O}$ (rango 13-18). Sin embargo, la VVS fue $20 \%$ (rango 17-26).

\section{Discusión}

Se observó en todos los casos que la volemia no era óptima luego de la resucitación habitual. Mientras que los objetivos de PVC, FC, TAM estaban dentro de los parámetros óptimos, la VVS sugería hipovolemia.

En relación a la evolución posterior de estos pacientes, uno fue de alta a los 60 días de internación, el segundo fallece a los 30 días por choque séptico a punto de partida pulmonar y el tercero también fallece a los 20 días por choque séptico a punta de partida pulmonar y abdominal.

Se concluye que es de gran utilidad contar con parámetros dinámicos para monitorizar la resucitación en los pacientes sépticos pero se requieren más estudios para corroborar estos hallazgos.

\section{Referencias bibliográficas}

1. Cannesson M, Musard H, Desebbe O, Boucau C, Simon R, Hénaine R, Lehot JJ. The ability of stroke volume variations obtained with Vigileo/FloTrac system to monitor fluid responsiveness in mechanically ventilated patients. Anesth Analg. 2009 Feb; 108(2): 513-7.

2. Benes J, Chytra I, Altmann P, Hluchy M, Kasal E, Svitak R, Pradl R, Stepan M. Intraoperative fluid optimization using stroke volume variation in high risk surgical patients: results of prospective randomized study. Crit Care. 2010; 14(3): R118.

3. Michard F. Changes in arterial pressure during mechanical ventilation. Anesthesiology 2005 Aug; 103(2): 419-428.

4. Durairaj L, Schmidt GA. Fluid therapy in resuscitated sepsis: less is more. Chest. 2008 Jan; 133(1): 252-63.

5. Rivers E, Nguyen B, Havstad S, Ressler J, Muzzin A, Knoblich B et al. Early goal-directed therapy in the treatment of severe sepsis and septic shock. N Engl J Med. 2001 Nov 8; 345(19): 1368-77.

6. Monnet X, Guérin L, Jozwiak M, Bataille A, Julien F, Richard C, Teboul JL. Pleth variability index is a weak predictor of fluid responsiveness in patients receiving norepinephrine. Br J Anaesth. 2013 Feb; 110(2): 207-13.

7. Monnet X, Anguel N, Jozwiak M, Richard C, Teboul JL. Third-generation FloTrac/Vigileo does not reliably track changes in cardiac output induced by norepinephrine in critically ill patients. Br J Anaesth. 2012 Apr; 108(4): 615-22. 